\title{
3D Hierarchically Scaffolds for Bone Repair: At the Crossroads of Experimental and Computational Outlooks
}

\author{
Paula Messina ${ }^{1}$ and Juan M. Ruso ${ }^{2, *}$ \\ 1 Department of Chemistry, INQUISUR-CONICET, Universidad Nacional del Sur, 8000 Bahía \\ Blanca, Argentina; E-Mail: pmessina@uns.edu.ar \\ 2 Soft Matter and Molecular Biophysics Group, Department of Applied Physics, University of \\ Santiago de Compostela, Santiago de Compostela E-15782, Spain. \\ * Author to whom correspondence should be addressed; E-Mail: juanm.ruso@usc.es \\ Tel.: +34- 881-811-000 (ext. 14042); Fax: +34-881-141-112.
}

Published: 4 December 2015

\begin{abstract}
A combination of experimental and theoretical approaches is proposed to determine the best template and conditions to synthesized a bio-active open mesopore structure material with a 3Dsponge-like network similar than those existed in trabecular bone with a consequent saving of time and products. Specifically, we discuss the possibility of combining experimental methods with theoretical methods that have been published in J Mater Sci 2012, 47, 2837-2844 and Langmuir 2015, doi: 10.1021/acs.langmuir.5b03074, respectively. The strategy proposed opens a new gate to the rational design of 3D hierarchically scaffolds for bone repair in the future.
\end{abstract}

Keywords: Self-Assembly; Scaffolds; QSPR; Biomaterials; Biomineralization; Bones; Biomaterials; Nanoparticles.

\section{Introduction}

Nanomedicine goes beyond the use of classical medicine at cell scales, currently faces an exceptional opportunity to improve global healthcare[1,2]. One of the main goals of nanomedicine is to develop new biocompatible scaffolds[3]. These materials in order to perform effectively must have an appreciation of the complex interrelationships between humans and the physical/chemical components of the environment [4].

Around $45 \%$ of the population aged 50 years and older will have an osteoporosis-related fracture in their lifetime. Such statistics demonstrate the vast need for new developments in this area. Bone is a complex composite material, with living and nonliving matter. The stiff nature of bone clearly enables it to form a semirigid framework, enable motion, and provide organ protection. Because of the trabecular bone exhibit spongelike bicontinuity at the millimeter scale, there is a growing interest in the synthesis of spongy-like materials[5]. However, there are too many factors to consider: immune response, vascularization, chemotactic and so on [6]. To overcome these challenges particular interest must be paid in the molecular sieve [7]. Here we propose a combination of experimental and computational tools to characterize and optimize the properties of sieves based on bicontinuous 
pore silica materials templated with bile salts mixtures.

\section{Results and Discussion}

Self-aggregation of binary systems has received considerable attention recently due to their potential as sustainable templates. In different works, we have studied, both experimentally and with molecular modeling, the optimization of the conditions and routes for the development of the best bio-active implantable materials. Such studies allows for the direct assessment of the role that molecular architecture and experimental conditions plays in approaches to simulate the trabecular bone organization

\subsection{Computational models}

On one side, in a previous work published this year we have built the first model that combines perturbation theories (PT) and linear free energy relationships (LFER) ideas [8]. The model uses as input covariance PT operators (CPTOs). CPTOs are calculated as the difference in covariance $\Delta \operatorname{Cov}\left({ }^{\mathrm{i}} \mu_{\mathrm{k}}\right)$ functions before and after multiple perturbations in the binary system[9]. In turn, we can calculate the covariances calculated as the product of two Box-Jenkins operators (BJO) operators [10]. BJOs are used to measure the deviation of the structure of different chemical compounds from a set of molecules measured in a given subset of experimental conditions $\left(b_{j}\right)$. The best CPT-LFER model found predicted the effects of 25000 perturbations over 9 different properties of binary systems. The best CPT-LFER model found with this algorithm was the following:

$$
\begin{gathered}
{ }^{0} f\left({ }^{p} \varepsilon_{i j}\right)_{\text {new }}=-0.275152{ }^{1} f\left({ }^{p} \varepsilon_{i j}\right)_{\text {ref }}- \\
-0.158186 \Gamma_{p i}(\text { dip })^{0}+0.037112 \Gamma_{p i}(\text { solv })^{0}+ \\
+0.017595 \Gamma_{p i}(\text { part })^{0}-0.150110 \Gamma_{p i}(\text { solv })^{-}+ \\
+0.095564 \Gamma_{p i}(\text { solv })^{+}+0.181357
\end{gathered}
$$

where the output function ${ }^{0} f\left({ }^{p} \varepsilon_{i j}\right)_{n e w}$ is a multi-output function that quantifies the numerical values $(\varepsilon)$ of different $p$ th physicochemical properties of the $i$ th binary system that have been experimentally determined under a certain set of $j$ th boundary conditions $\left(\mathrm{c}_{\mathrm{j}}\right) . \Gamma_{j p}(k)^{q}$ are the covariance perturbation functions. The types of potentials were the electrostatic dipole potential (dip), electrostatic potential in solution (solv) and thermodynamic potential for water-nonpolar phase partitioning (part). The notation for $\mathrm{q}$ is 0 when both species are molecules, + and - when both species are cations and anions, respectively.

\subsection{Experimental methods}

On the other hand, in other recent work [11] we reported the experimental study of the physicochemical properties of the nanostructures. The nanostructures studied are formed within aqueous mixtures of bile salts sodium glycodeoxycholate (NaGDC), dehydrocholic acid (HDHC), Sodium deoxycholate (NaDC) and surfactant didodecyldime thylammonium bromide (DDAB) as a function of total concentration and mixed ratio. The experimental study has been carried out by means of thermodynamic analysis, fluorescence spectroscopy, field emission-scanning electron microscopy (FE-SEM) and energy dispersive Xray microanalysis (EDX) experiments [11].

Experimental measurements were perfectly reproduced by our CPT-LFER model, which was further used to examine different systems under other conditions not studied experimentally with a consequent saving of time and products.

The siliceous materials (SM) were prepared using hydrothermal synthesis. Tetraethyl orthosilicate (TEOS) was added to different bile salts mixture solutions. The mixture was stirred and left for $24 \mathrm{~h}$ in an autoclave at $100{ }^{\circ} \mathrm{C}$. The obtained materials were calcined [12]. The material final structures take place through a vesicle to sponge-like phase transformation whose driving force seems to be the interaction of bile salts and silica species during the material polymerization synthesis step. Depending of the type and amount of BS in the template mixture, the film can curve toward the a-polar or toward the polar side leading to different final morphologies. The highly hydrophobic steroid backbone of $\mathrm{NaDHC}$ molecule causes a great disturbance in the templated liquid crystal mixture. The final mesophases obtained depending on the materials arises in different structures.

It was demonstrated that the requirement for an artificial material to bond to living bone (bioactivity) is the formation of bonelike apatite 
(HA) on its surface when implanted in the living body [13],[14], and that this in vivo apatite formation can be reproduced in a SBF with ion concentrations nearly equal to those of human blood plasma.

The existence of a highly porous surface can accelerate the biomimetic process. In agreements, the apatite deposition was observed only in such specimens which content mesopores and high proportion of siloxane bridges in their structures. The time evolution of apatite growth on the synthesized materials was followed by FT-IR measurements (ESM) and confirmed by scanning electron microscopy. Figure 1 show the FE-SEM microphotographs of the material surfaces after soaking in 1.5 SBF. It must be noticed that calcium phosphate coatings grow not only on the material surface but also in the pore interior and the progress of this covering increases as a function of the soaking time SEM revealed the presence of preformed calcium phosphate coatings to be composed entirely of straight platelike units with sharp edges, with a change in crystal geometry as the time of soaking increased. The definite crystalline structure is achieved after soaking for 20 days in SBF. The thickness of the apatite-like coating increases with time and reaches a saturated point after 10 days of soaking. Assuming that the growth rate of apatite coating is controlled by the calcium and phosphorous ions diffusion rates from the SBF to the material surface, the growth kinetics of apatite-like coatings on porous materials can be expressed by an empirical relationship

$$
d^{2}=K t
$$

where $d$ is the thickness of the coating evaluated from SEM photos, $t$ is the soaking time for the biomimetic deposition, and $\mathrm{K}$ is the growth rate constant. The growth rate constant obtained was, $\mathrm{K}=2.02089 \times 10^{-18} \mathrm{~m}^{2} \mathrm{seg}^{-1}$.
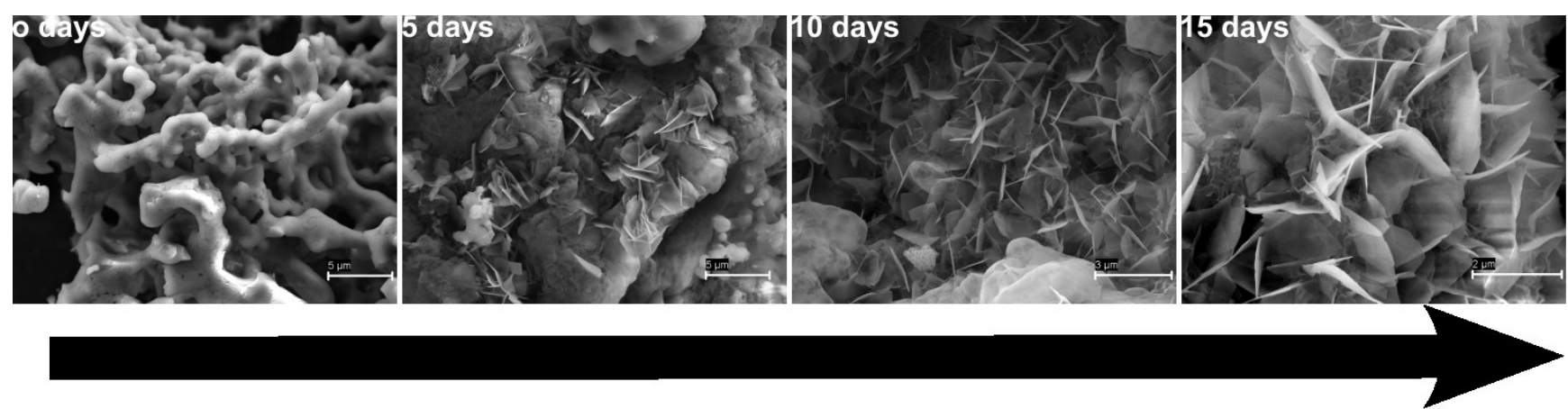

Figure 1. FE-SEM microphotographs showing the time evolution of HA layer formation on materials. Scale bars $3-5 \mu \mathrm{m}$.

\section{Materials and Methods}

Dihidroxy bile salt sodium glycodeoxycholate (NaGDC), dehydrocholic acid (HDHC), sodium deoxycholate (NaDC), didodecyldimethyl ammonium bromide (DDAB) and tetraethyl orthosilicate (TEOS) were purchased from Sigma-Aldrich and used as received.

Fluorescence Spectroscopy. Fluorescence spectra of pyrene were obtained with a Cary Eclipse spectrophotometer equipped with a temperaturecontrol device and a multicell sample holder (Varian 198 Instruments Inc.). All samples were

prepared with saturated solutions of pyrene $\left(3 \times 10^{-7} \mathrm{~mol} \mathrm{dm}^{-3}\right)$. Measurements were performed at $298 \mathrm{~K}$, and the fluorescence intensities ratios (I1/I3) of the first (I1, $373 \mathrm{~nm}$ ) and third (I3, $384 \mathrm{~nm})$ peaks from the short wavelength in the spectra of pyrene were obtained with excitation at $\lambda=335 \mathrm{~nm}$. The excitation and emission slit widths were set to be 5 and $1.5 \mathrm{~nm}$, respectively.

Field emission-scanning electron microscopy (FE-SEM) and energy dispersive X-ray microanalysis (EDX) were performed using a 
FE-SEM ULTRA PLUS microscope. Microanalysis EDX: resolution $129 \mathrm{eV}$ and wavelength-dispersive (WD) $8.5 \mathrm{~nm}$. Transmission electron microscopy (TEM) was performed using a Philips CM-12 transmission electron microscope equipped with a digital camera MEGA VIEWII DOCU.

Infrared spectra were collected using a NicoletNexus $470 \quad$ Fourier-Transform infrared Spectrometer (FT-IR) equipped with a pneumatic motion interferometer.

\section{Conclusions}

We successfully presented a new computational method for predicting key physicochemical properties of compounds for biomaterials templating. For this purpose we developed the first model which combines perturbation theory (PT) and linear free energy relationship (LFER) ideas. We show that a single model may have a good quality of predicting multiple properties. Results obtained by our model were compared with published data, and our experimental results obtained standard errors less than $0.02 \%$.

We also demonstrated that controlling molecular architecture is an efficient platform to manipulate the material bioactivity. The presence of siloxane bridges and its distribution results in HA growth not only at the surface but also at pore interior. Finally, the kinetics of HA formation was also tested and we concluded that the definite crystalline structure is achieved after 20 days.

The detailed analysis and theoretical model provided in these studies is expected to be useful as a reference to design in a fast and economical way new bioactive scaffolds for bone regeneration based on surfactant mixtures.

\section{Acknowledgments}

Authors acknowledge Fundación Ramón Areces, Universidad Nacional del Sur (PGI 24/Q064), and Concejo Nacional de Investigaciones Cientifícas y Técnicas de la República Argentina (CONICET, PIP-11220130100100CO) for their financial support.

\section{Author Contributions}

All authors have contributed equally to this work.

\section{Conflicts of Interest}

The authors declare no conflict of interest.

\section{References and Notes}

1. Schillmeier, M. Caring for social complexity in nanomedicine. Nanomedicine 2015, 10, 31813193.

2. Ruso, J.M.; Deo, N.; Somasundaran, P. Complexation between dodecyl sulfate surfactant and zein protein in solution. Langmuir 2004, 20, 8988-8991.

3. V. Messina, P.; Miguel Besada-Porto, J.; M. Ruso, J. Self-assembly drugs: From micelles to nanomedicine. Current Topics in Medicinal Chemistry 2014, 14, 555-571.

4. $\quad$ Fu, S.; Ni, P.; Wang, B.; Chu, B.; Peng, J.; Zheng, L.; Zhao, X.; Luo, F.; Wei, Y.; Qian, Z. In vivo biocompatibility and osteogenesis of electrospun poly( $\varepsilon$-caprolactone)-poly(ethylene glycol)-poly(e-caprolactone)/nano-hydroxyapatite composite scaffold. Biomaterials 2012, 33, 8363-8371.

5. M. Ruso, J.; Sartuqui, J.; V. Messina, P. Multiscale inorganic hierarchically materials: Towards an improved orthopaedic regenerative medicine. Current Topics in Medicinal Chemistry 2015, 15, 2290-2305.

6. Sartuqui, J.; D' Elía, N.; Gravina, A.N.; Messina, P.V. Analyzing the hydrodynamic and crowding evolution of aqueous hydroxyapatite-gelatin networks: Digging deeper into bone scaffold design variables. Biopolymers 2015, 103, 393-405. 
7. Ruso, J.M.; Pardo, V.; Sartuqui, J.; Gravina, N.; D’Elía, N.L.; Pieroni, O.I.; Messina, P.V. Photoluminescent sba-16 rhombic dodecahedral particles: Assembly, characterization, and ab initio modeling. ACS Applied Materials \& Interfaces 2015, 7, 12740-12750.

8. Messina, P.V.; Besada-Porto, J.M.; González-Díaz, H.; Ruso, J.M. Self-assembled binary nanoscale systems: Multioutput model with lfer-covariance perturbation theory and an experimental-computational study of nagdc-ddab micelles. Langmuir 2015.

9. Casañola-Martin, G.; Le-Thi-Thu, H.; Pérez-Giménez, F.; Marrero-Ponce, Y.; Merino-Sanjuán, M.; Abad, C.; González-Díaz, H. Multi-output model with box-jenkins operators of linear indices to predict multi-target inhibitors of ubiquitin-proteasome pathway. Mol Divers 2015, 19, 347-356.

10. Casañola-Martin, G.M.; Le-Thi-Thu, H.; Pérez-Giménez, F.; Ponce, Y.M.; Sanjuán, M.M.; Abad, C.; Díaz, H.G. Multi-output model with box-jenkins operators of quadratic indices for prediction of malaria and cancer inhibitors targeting ubiquitin-proteasome pathway (upp) proteins. Curr Protein Pept Sci 2015.

11. Fernández-Leyes, M.; Verdinelli, V.; Hassan, N.; Ruso, J.; Pieroni, O.; Schulz, P.; Messina, P. Biomimetic formation of crystalline bone-like apatite layers on spongy materials templated by bile salts aggregates. J Mater Sci 2012, 47, 2837-2844.

12. Ruso, J.M.; Verdinelli, V.; Hassan, N.; Pieroni, O.; Messina, P.V. Enhancing cap biomimetic growth on tio2 cuboids nanoparticles via highly reactive facets. Langmuir 2013, 29, 23502358 .

13. Hassan, N.; Verdinelli, V.; Ruso, J.M.; Messina, P.V. Mimicking natural fibrous structures of opals by means of a microemulsion-mediated hydrothermal method. Langmuir 2011, 27, 89058912.

14. Gravina, N.; Ruso, J.M.; Mbeh, D.A.; Yahia, L.H.; Merhi, Y.; Sartuqui, J.; Messina, P.V. Effect of ceria on the organization and bio-ability of anatase fullerene-like crystals. RSC Advances 2015, 5, 8077-8087.

(C) 2015 by the authors; licensee MDPI, Basel, Switzerland. This article is an open access article distributed under the terms and conditions defined by MDPI AG, the publisher of the Sciforum.net platform. Sciforum papers authors the copyright to their scholarly works. Hence, by submitting a paper to this conference, you retain the copyright, but you grant MDPI AG the non-exclusive and unrevocable license right to publish this paper online on the Sciforum.net platform. This means you can easily submit your paper to any scientific journal at a later stage and transfer the copyright to its publisher (if required by that publisher). (http://sciforum.net/about ). 